\title{
Athletes feature greater rates of muscle glucose transport and glycogen synthesis during lipid infusion
}

\author{
Esther Phielix, ${ }^{1,2,3}$ Paul Begovatz, ${ }^{1,2}$ Sofiya Gancheva, ${ }^{1,2,4}$ Alessandra Bierwagen, ${ }^{1,2}$ Esther Kornips, ${ }^{3}$ \\ Gert Schaart, ${ }^{3}$ Matthijs K. C. Hesselink, ${ }^{3}$ Patrick Schrauwen, ${ }^{3}$ and Michael Roden ${ }^{1,2,4}$ \\ IInstitute for Clinical Diabetology, German Diabetes Center, Düsseldorf, Germany. 'erman Center for Diabetes Research, \\ München-Neuherberg, Germany. ${ }^{3}$ Department of Nutrition and Movement Sciences, School for Nutrition and Translational \\ Research in Metabolism, Maastricht University Medical Center, Maastricht, Netherlands. ${ }^{4}$ Division of Endocrinology and \\ Diabetology, Medical Faculty, Heinrich-Heine University, Düsseldorf, Germany.
}

BACKGROUND. Insulin resistance results from impaired skeletal muscle glucose transport/ phosphorylation, linked to augmented lipid availability. Despite greater intramuscular lipids, athletes are highly insulin sensitive, which could result from higher rates of insulin-stimulated glycogen synthesis or glucose transport/phosphorylation and oxidation. Thus, we examined the time course of muscle glycogen and glucose-6-phosphate concentrations during low and high systemic lipid availability.

METHODS. Eight endurance-trained and 9 sedentary humans ( $\left(\mathrm{VO}_{2}\right.$ peak: $56 \pm 2$ vs. $33 \pm 2 \mathrm{~mL}$ ) $\mathrm{kg} / \mathrm{min}, P<0.05$ ) underwent 6 -hour hyperinsulinemic-isoglycemic clamp tests with infusions of triglycerides or saline in a randomized crossover design. Glycogen and glucose-6-phosphate concentrations were monitored in vastus lateralis muscles using ${ }^{13} \mathrm{C} /{ }^{31} \mathrm{P}$ magnetic resonance spectroscopy.

RESULTS. Athletes displayed a $25 \%$ greater $(P<0.05)$ insulin-stimulated glucose disposal rate $(R d)$ than sedentary participants. During Intralipid infusion, insulin sensitivity remained higher in the athletes ( $\Delta \mathrm{Rd}$ : $25 \pm 3$ vs. $17 \pm 3 \mu \mathrm{mol} / \mathrm{kg} / \mathrm{min}, P<0.05$ ), supported by higher glucose transporter type 4 protein expression than in sedentary humans. Compared to saline infusion, AUC of glucose6-phosphate remained unchanged during Intralipid infusion in athletes $(1.6 \pm 0.2 \mathrm{mmol} / \mathrm{L}$ vs. 1.4 $\pm 0.2[\mathrm{mmol} / \mathrm{L}] \times \mathrm{h}, P=$ n.s. $)$ but tended to decrease by $36 \%$ in sedentary humans $(1.7 \pm 0.4 \mathrm{vs} .1 .1$ $\pm 0.1[\mathrm{mmol} / \mathrm{L}] \times \mathrm{h}, P<0.059)$. This drop was accompanied by a $72 \%$ higher rate of net glycogen synthesis in the athletes upon Intralipid infusion ( $47 \pm 9 \mathrm{vs.} 13 \pm 3 \mu \mathrm{mol} / \mathrm{kg} / \mathrm{min}, P<0.05$ ).

CONCLUSION. Athletes feature higher skeletal muscle glucose disposal and glycogen synthesis during increased lipid availability, which primarily results from maintained insulin-stimulated glucose transport with increased myocellular glucose-6-phosphate levels for subsequent glycogen synthesis.

TRIAL REGISTRATION. ClinicalTrials.gov NCT01229059.

Authorship note: $\mathrm{EP}, \mathrm{PB}$, and SG contributed equally to the work.

Conflict of interest: The authors have declared that no conflict of interest exists.

Copyright: (c) 2019, American Society for Clinical Investigation.

Submitted: February 4, 2019 Accepted: October 2, 2019 Published: November 1, 2019.

Reference information: /CI Insight. 2019:4(21):e127928. https://doi.org/10.1172/jci. insight.127928.
FUNDING. German Federal Ministry of Health (BMG).

\section{Introduction}

Insulin resistance characterizes obesity, type 2 diabetes (T2DM) (1), and nonalcoholic fatty liver disease (2) and strongly correlates with intramyocellular lipid content (IMLC) in sedentary individuals (3-5). Likewise, elevation of circulating free fatty acids rapidly induces peripheral insulin resistance in sedentary humans (6-9). Using noninvasive monitoring of muscle glucose-6-phosphate (G6P) and glycogen concentrations with ${ }^{31} \mathrm{P} /{ }^{13} \mathrm{C}$ magnetic resonance spectroscopy (MRS), we demonstrated that lipid-induced insulin resistance is preceded by attenuated increases in muscle G6P reflecting inhibition of glucose transport/ phosphorylation in sedentary humans $(9,10)$. This is followed by slower rates of nonoxidative glucose 
disposal (NOGD) reflecting lower muscle glycogen synthesis. Similar reductions in muscle glucose transport/phosphorylation are typical for chronic insulin-resistant states such as obesity and T2DM (11-13).

In contrast to sedentary humans, endurance-trained athletes generally present with higher IMLC content in the face of higher peripheral insulin sensitivity (14). We previously reported that endurance-trained insulin-sensitive humans could be at least partially protected from lipid-induced insulin resistance (15). Upon lipid infusion, these athletes exhibited an only minor decrease in peripheral insulin sensitivity compared to untrained humans. However, this study did not allow us to clarify whether greater muscle glucose transport/ phosphorylation or glycogen synthesis is mainly responsible for the protection from lipid-induced insulin resistance in trained skeletal muscle. Noninvasive ${ }^{13} \mathrm{C}$ MRS is the state-of-the-art technique to directly monitor the time course of intramuscular G6P and glycogen concentrations and to measure net rates of muscle glycogen synthesis. This method overcomes artifacts resulting from ex vivo glycogen breakdown in muscle biopsies; from steady state issues and extramuscular glucose metabolism, which affect both glucose disappearance rate $(\mathrm{Rd})$ and glucose appearance rate $(\mathrm{Ra})$ in isotopic dilution studies; and from the intraindividual variability of indirect calorimetry, which affects the assessment of nonoxidative glucose metabolism (16).

Skeletal muscle glucose uptake is regulated by glucose transporter type 4 (GLUT4) abundance as well as by translocation from the cytosol toward the sarcolemmal membrane, which is independently stimulated by exercise and insulin. The insulin-mediated translocation of GLUT4 to the sarcolemmal membrane is impaired in patients with T2DM $(17,18)$ and results in lower intramuscular glucose and in turn lower G6P concentrations (19). Intravenous intralipid infusion reduces both intramuscular glucose and G6P concentrations, preceding the development of lipid-induced insulin resistance (20). Total GLUT4 protein and GLUT4 mRNA content also rise over time with chronic endurance training (21-23) as well as within 3 hours of exercising (24). This may potentiate the higher insulin-stimulated glucose uptake and glycogen storage in skeletal muscle after exercise. Interestingly, a single session of exercise preceding intralipid infusion completely prevented lipid-induced insulin resistance (25). This could partly be explained by postexercise high insulin-stimulated IRS-1-associated PI3K activity, responsible for GLUT4 translocation (25). Several studies provided evidence for a link between acute lipid-induced insulin resistance and defective insulin signaling (26-29). Moreover, a recent study indicated that activation of the DAG/protein kinase C- $\theta$ (PKC- $\theta$ ) pathway during intralipid infusion inhibits muscle insulin signaling in sedentary humans (30); however, its role in athletes remains controversial (31). To our knowledge, no studies reported on GLUT4 protein levels in the vicinity of increased free fatty acids via the infusion of Intralipid in sedentary individuals or in endurance athletes.

This study therefore tested the hypothesis that endurance-trained athletes maintain greater rates of insulin-stimulated muscle glycogen synthesis in vivo during lipid infusion than sedentary humans. In addition, this study examined whether glucose transport/phosphorylation is rate controlling in athletes under insulin-stimulated conditions.

\section{Results}

Participants' characteristics. By design, athletes had higher $\mathrm{VO}_{2}$ peak values (Table 1). Athletes also showed slightly lower fasting plasma insulin concentrations $(P=0.07)$. Fasting plasma glucose, triglycerides (TGs), free fatty acids (FFAs), and glycerol concentrations at baseline were comparable between groups (Table 1). Fasting glucagon concentrations were not different between athletes and sedentary participants (Table 1).

Plasma lipids. During saline infusion, insulin similarly suppressed circulating FFA concentrations in both groups (Figure 1A), while plasma FFA concentrations increased in both groups during the clamp with Intralipid infusion, with a tendency toward higher FFA levels in sedentary participants at the end of the infusion period $(P$ $=0.07$ ) (Figure 1B). Serum TGs (32) increased upon Intralipid versus saline infusion, with no difference between athletes and sedentary participants (TG during Intralipid $402 \pm 66 \mathrm{mg} / \mathrm{dL}$ in sedentary and $312 \pm 49 \mathrm{mg} / \mathrm{dL}$ in athletes vs. TG during saline $48 \pm 9 \mathrm{mg} / \mathrm{dL}$ in sedentary vs. $35 \pm 6 \mathrm{mg} / \mathrm{dL}$ in athletes; $P<0.05$ for Intralipid vs. saline; $P=$ n.s. between sedentary humans and athletes). Likewise, plasma glycerol concentrations were higher upon Intralipid versus saline infusion without a difference between groups (glycerol during Intralipid $76 \pm 11$ $\mathrm{mmol} / \mathrm{L}$ in sedentary and $62 \pm 11 \mathrm{mmol} / \mathrm{L}$ in athletes vs. glycerol during saline $14 \pm 1 \mathrm{mmol} / \mathrm{L}$ in sedentary and $15 \pm 1 \mathrm{mmol} / \mathrm{L}$ in athletes; $P<0.05$ for Intralipid vs. saline; $P=$ n.s. between sedentary and athletes).

Whole-body and hepatic glucose metabolism. On saline infusion, insulin-stimulated $\Delta \mathrm{Rd}$ was higher in athletes than in sedentary participants at $t=210-240$ minutes (Figure 1C) and $t=330-360$ minutes (Figure 1D, both $P<0.05$ ). Upon Intralipid infusions, $\Delta \mathrm{Rd}$ dropped in athletes as well as in sedentary 
Table 1. Participants' characteristics

\begin{tabular}{|c|c|c|}
\hline & Sedentary $(n=9)$ & Athletes $(n=8)$ \\
\hline Age (yr) & $23.4 \pm 1.1$ & $26.6 \pm 1.4$ \\
\hline Body weight (kg) & $71.7 \pm 3.0$ & $68.7 \pm 3.3$ \\
\hline $\mathrm{VO}_{2}$ peak $(\mathrm{mL} / \mathrm{kg} / \mathrm{min})$ & $33.2 \pm 1.9$ & $56.0 \pm 1.5^{A}$ \\
\hline Fasting glucose (mmol/L) & $4.2 \pm 0.1$ & $4.1 \pm 0.1$ \\
\hline Fasting insulin (mU/L) & $6.3 \pm 1.1$ & $4.6 \pm 0.5$ \\
\hline Fasting glycerol (mmol/L) & $23 \pm 1$ & $22 \pm 2$ \\
\hline Fasting glucagon (pg/mL) & $83 \pm 13$ & $105 \pm 17$ \\
\hline
\end{tabular}

Data are mean \pm SEM. ${ }^{A} P<0.05$ vs. sedentary participants.

participants at $t=210-240$ minutes (Figure 1C) and $t=330-360$ minutes (both $P<0.05$ ) (Figure 1D). At 330-360 minutes of Intralipid infusion, $\Delta \mathrm{Rd}$ was approximately $57 \%$ and approximately $62 \%$ lower (both $P<0.05$ ) in athletes and sedentary participants, respectively. Average baseline rates for endogenous glucose production (EGP) (before the start of infusions) were slightly higher in athletes compared with sedentary participants $(13.2 \pm 0.5 \mu \mathrm{mol} / \mathrm{kg} / \mathrm{min}$ vs. $11.8 \pm 0.6 \mu \mathrm{mol} / \mathrm{kg} / \mathrm{min}, P<0.05)$. At 210 240 minutes Intralipid infusion, hepatic insulin sensitivity was similar between sedentary participants and athletes $(P=$ n.s., Figure 1E), but suppression of EGP was higher in athletes at 330-360 minutes ( $-76 \%$ vs. $-63 \%$ in sedentary, $P<0.05$, Figure $1 \mathrm{~F})$. Within sedentary participants, at $330-360$ minutes, hepatic insulin sensitivity was also lower during Intralipid versus saline condition (-63\% vs. $-96 \%, P<$ 0.05 , Figure $1 \mathrm{~F})$. At this time, hepatic insulin sensitivity remained comparable between both conditions for athletes $(-76 \%$ vs. $-86 \%, P=$ n.s., Figure $1 \mathrm{~F})$.

Time course of muscle glucose flux. The time course of the glucose infusion rates (GIRs) throughout saline and Intralipid infusion studies in sedentary participants and in athletes is shown in Figure 2, A and B, respectively. The AUC of GIR was about 30\% higher under saline-infused conditions in athletes $(0.99 \pm$ $0.06 \mu \mathrm{mol} / \mathrm{kg})$ than in sedentary participants $(0.70 \pm 0.06 \mu \mathrm{mol} / \mathrm{kg}, P<0.05$, Figure $2 \mathrm{C})$.

To examine tissue-specific glucose flux in skeletal muscle, we monitored in vivo glycogen and G6P concentrations. At baseline, average muscle glycogen concentration was 2-fold higher in athletes compared with sedentary participants $(110 \pm 17$ vs. $54 \pm 2 \mathrm{mmol} / \mathrm{L}, P<0.05)$. The time course of insulin-stimulated glycogen concentrations, given by the difference between the clamp and baseline $(\Delta \mathrm{gly}$ cogen), were monitored for sedentary participants (Figure 2D) and athletes (Figure 2E). During saline conditions, the AUC of $\Delta$ glycogen was higher in athletes $(92 \pm 13[\mathrm{mmol} / \mathrm{L}] \times \mathrm{h})$ than in sedentary participants $(46 \pm 8[\mathrm{mmol} / \mathrm{L}] \times \mathrm{h}, P<0.01$, Figure $2 \mathrm{E})$. During Intralipid infusion, the AUC of $\Delta \mathrm{gly}-$ cogen was higher in the athletes $(69 \pm 14[\mathrm{mmol} / \mathrm{L}] \times \mathrm{h})$ compared with the sedentary participants $(24$ $\pm 5[\mathrm{mmol} / \mathrm{L}] \times \mathrm{h}, P<0.01$, Figure $2 \mathrm{~F})$. Within the athletes, the AUC was only nominally, but not significantly $(P=0.23)$, lower upon Intralipid than upon saline infusion (Figure $2 \mathrm{~F})$. Within the group of sedentary participants, the AUC fell by approximately $49 \%$ upon Intralipid compared with saline infusion $(P<0.01)$. From the glycogen concentrations, we calculated the net muscle glycogen synthesis rate (GSR) for the time intervals 0-180 minutes and 180-360 minutes (Figure 3) as well as for the entire period (0-360 minutes). Initially (0-180 minutes), the GSR was approximately $64 \%$ higher in the athletes than in sedentary participants under saline conditions $(P<0.05)$ and approximately $72 \%$ higher under lipid-infused conditions $(P<0.05)$. From 180 minutes to 360 minutes, the GSR did not differ between athletes and sedentary participants under saline-infused conditions ( $P=$ n.s., Figure 3B). The GSR over the entire period from 0 to 360 minutes during saline infusion was approximately $46 \%$ higher in athletes versus sedentary participants $(P<0.001$, Figure 3C). However, Intralipid infusion completely inhibited GSR between 180 and 360 minutes in the sedentary participants $(P<0.05$, Figure $3 \mathrm{~B})$ in contrast to the athletes. The GSR calculated over the entire period (0-360 minutes) did not differ between Intralipid and saline infusion in the athletes $(P=$ n.s., Figure $3 C)$ but decreased by $72 \%(P<0.01$, Figure $3 C)$ in the sedentary participants during Intralipid compared with saline infusion. 
A

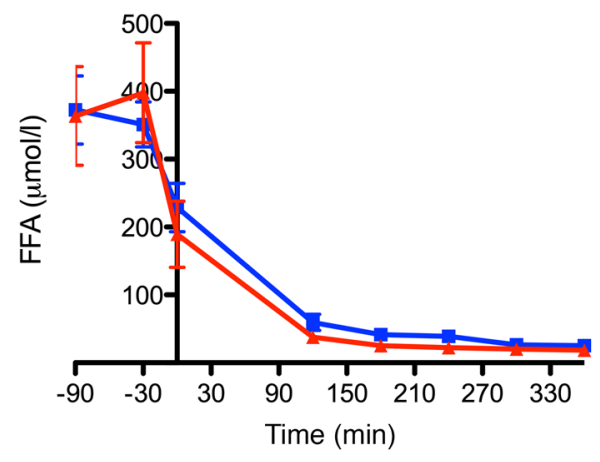

C

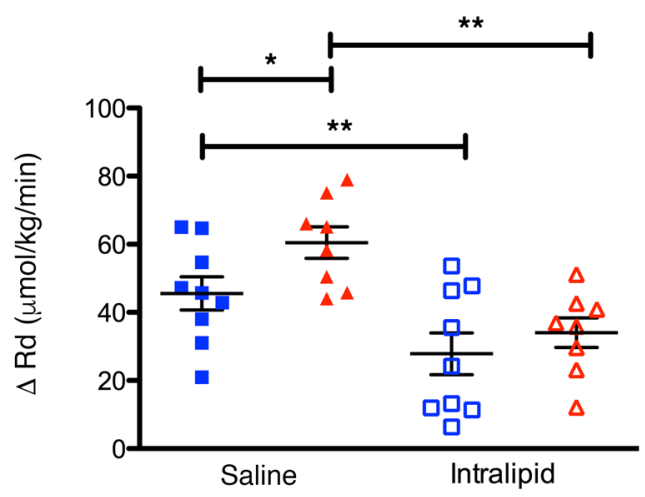

$\mathbf{E}$

E $210-240 \mathrm{~min}$

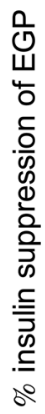

Saline

$210-240 \mathrm{~min}$
B

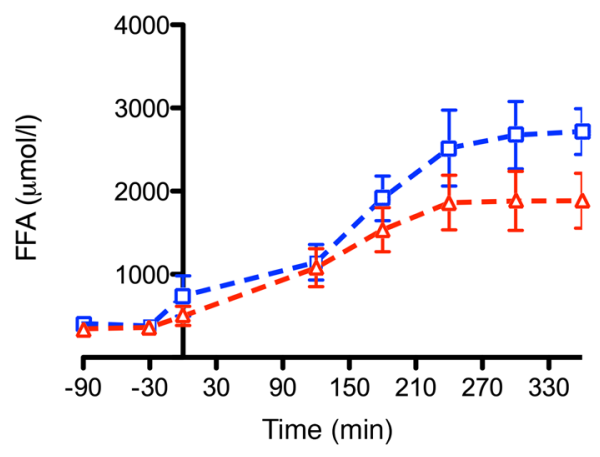

D

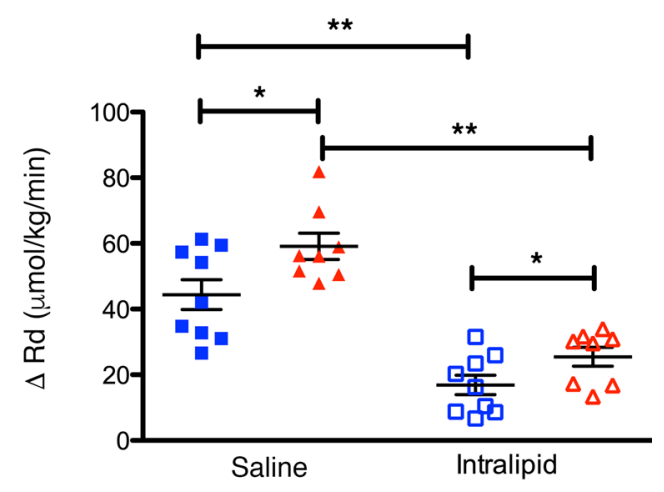

$\mathbf{F}$

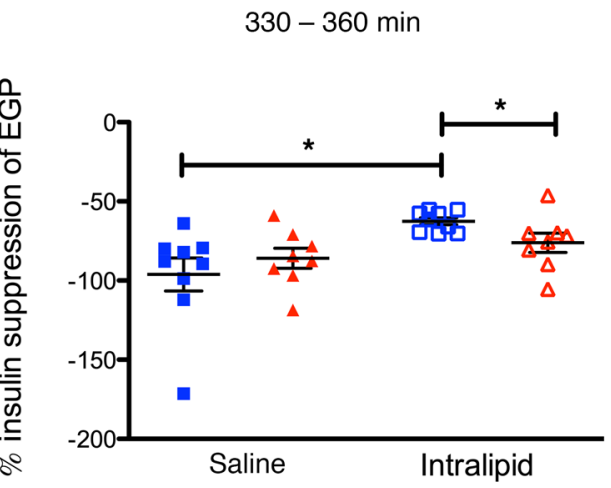

Figure 1. Plasma FFA and insulin sensitivity during saline or Intralipid infusion. Nine sedentary humans (blue squares) and 8 endurance-trained athletes (red triangles) underwent 2 hyperinsulinemic-isoglycemic clamp tests with either saline (full symbols and lines) or Intralipid infusion (empty symbols and dashed lines). The time course of plasma FFA ( $\mathbf{A}$ and $\mathbf{B}$ ) and whole-body (expressed as $\triangle$ Rd vs. baseline; $\mathbf{C}$ and $\mathbf{D}$ ) and hepatic (expressed as percentage of insulin-suppressed EGP vs. baseline; $\mathbf{E}$ and $\mathbf{F}$ ) insulin sensitivity is shown. Insulin sensitivity was calculated for 2 steady state time intervals, 210-240 minutes (C and $\mathbf{E}$ ) and 330-360 minutes ( $\mathbf{D}$ and $\mathbf{F}$ ). Data expressed as mean $\pm \mathrm{SEM} ;{ }^{*} P<0.05$ and ${ }^{* *} P<0.01$. FFA, free fatty acid; EGP, endogenous glucose production; $\Delta \mathrm{Rd}$, delta rate of glucose disappearance during insulin stimulation minus baseline.

The higher $\Delta$ glycogen and GSR of the athletes during Intralipid infusion were paralleled by the time course of intramyocellular G6P concentrations (Figure 2G). The AUC of G6P in athletes upon Intralipid infusion did not differ from the AUC of G6P concentrations during saline infusion (athletes: AUC Intralipid $1.4 \pm 0.2$ vs. AUC saline $1.6 \pm 0.2[\mathrm{mmol} / \mathrm{L}] \times \mathrm{h}, P=$ n.s., Figure $2 \mathrm{I})$. Conversely, sedentary participants showed a strong trend toward lower AUC of G6P during Intralipid than during saline infusion (sedentary: $1.1 \pm 0.1$ vs. $1.7 \pm 0.4[\mathrm{mmol} / \mathrm{L}] \times \mathrm{h}, P=0.059$, Figure $2 \mathrm{H}$ ). These data indicate that glucose transport is 
A

Athletes

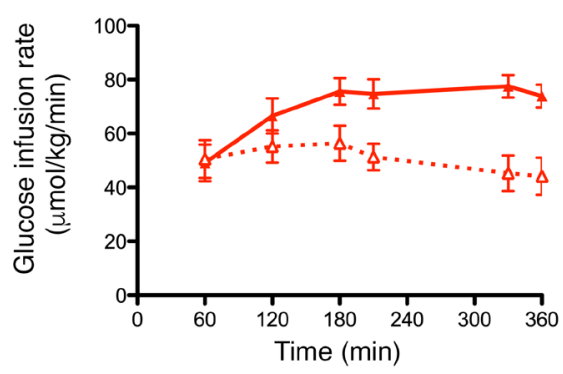

D

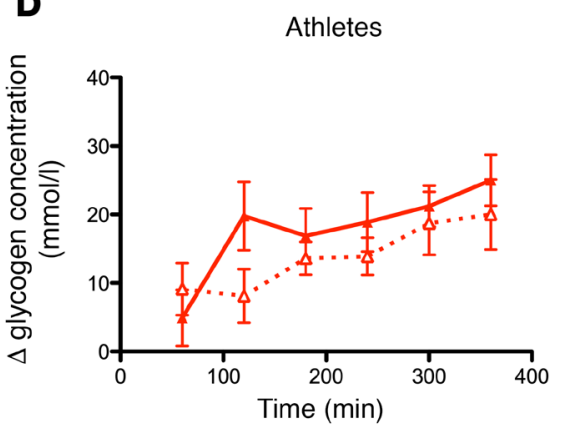

G

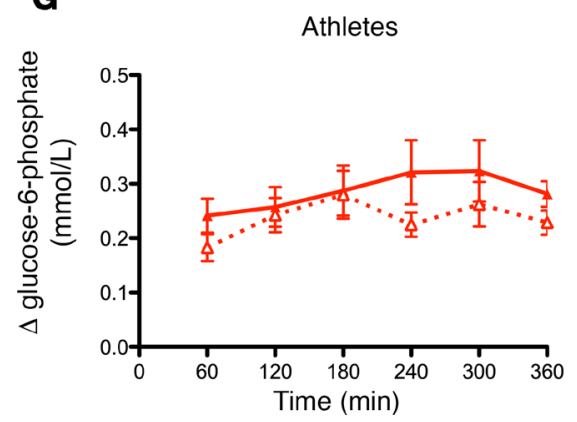

B

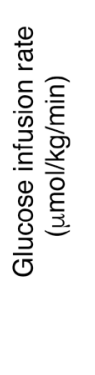

Sedentary

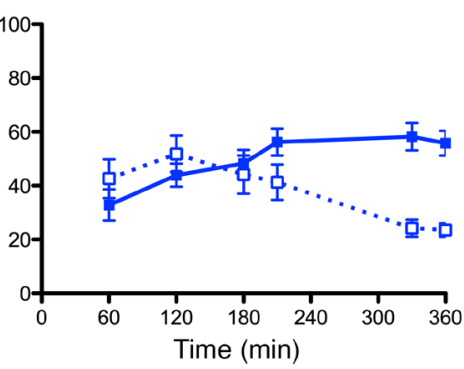

Sedentary

E

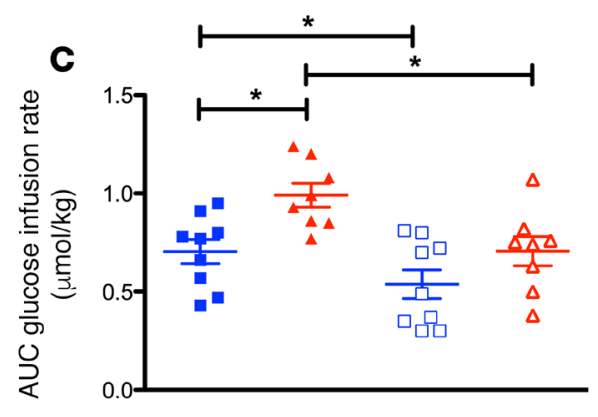

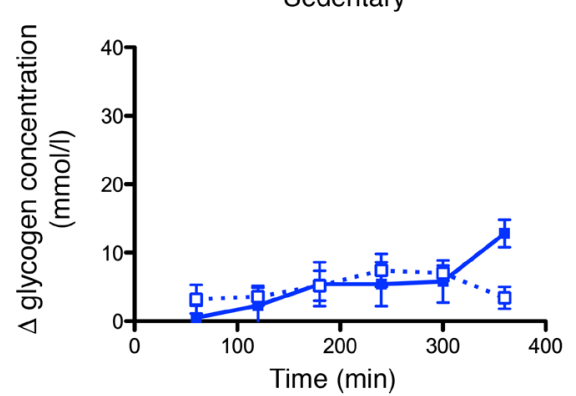

H

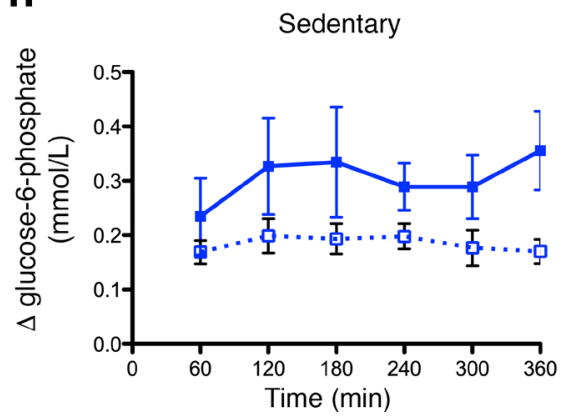

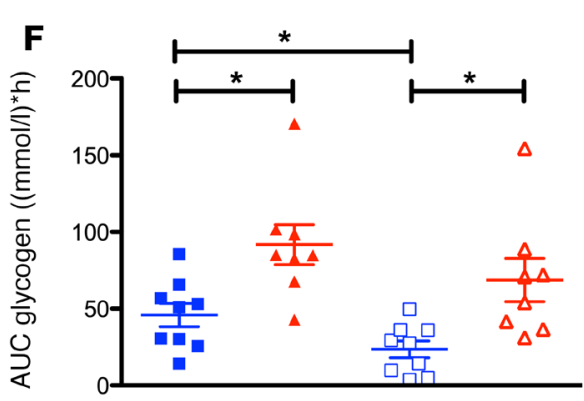

Figure 2. Time course of changes in GIRs, intramuscular glycogen, and intramuscular G6P during saline or Intralipid infusion. Nine sedentary humans (blue squares) and 8 endurance-trained athletes (red triangles) underwent 2 hyperinsulinemic-isoglycemic clamp tests with either saline (full symbols and lines) or Intralipid infusion (empty symbols and dashed lines). Infusion rates of exogenous glucose are shown for sedentary participants (A) and for athletes (B), as well as the AUC (C). Changes in insulin-stimulated muscle glycogen concentrations (measured with in vivo ${ }^{13} \mathrm{C}-\mathrm{MRS}$ and expressed as $\Delta$ glycogen vs. baseline) as well as the AUC (D-F) and G6P (measured with in vivo ${ }^{31} \mathrm{P}-\mathrm{MRS}$ and expressed as $\triangle \mathrm{G} 6 \mathrm{P}$ ) and the AUC (G-I) during the clamps are depicted. Data are expressed as mean $\pm \mathrm{SEM} ;{ }^{*} P<0.05$ and ${ }^{* *} P<0.01$.

reduced upon Intralipid infusion in sedentary participants but not in athletes. This reduction in glucose transport in sedentary people could result from reduced GSR. In athletes, however, the GSR did not account for reduced glucose transport during Intralipid infusion.

Within the athlete group, we performed a subgroup analysis of the differences of AUCs of $\Delta$ glycogen and $\Delta \mathrm{G} 6 \mathrm{P}$ between the saline- and Intralipid-infused conditions for runners $(n=5)$ compared with cyclers $(n=3)$. The AUC for $\Delta$ glycogen under saline versus Intralipid did not differ for runners (AUC $\Delta$ glycogen: saline $79 \pm 9$ vs. Intralipid $54 \pm 9$ [mmol/L] $\times$ h,$P=$ n.s. $)$ or for cyclers $(\Delta$ glycogen: saline $113 \pm 18$ vs. Intralipid $94 \pm 19[\mathrm{mmol} / \mathrm{L}] \times \mathrm{h}, P=\mathrm{n} . \mathrm{s})$. Also, for $\Delta \mathrm{G} 6 \mathrm{P}$ no differences were found between runners (AUC $\Delta$ G6P: saline $1.7 \pm 0.3$ vs. Intralipid $1.4 \pm 0.2[\mathrm{mmol} / \mathrm{L}] \times \mathrm{h}, P=$ n.s.) and cyclers (AUC $\Delta \mathrm{G} 6 \mathrm{P}$ : saline 1.5 \pm 0.1 vs. Intralipid $1.6 \pm 0.2[\mathrm{mmol} / \mathrm{L}] \times \mathrm{h}, P=\mathrm{n} . \mathrm{s}$.). Differences between runners and cyclers with the sedentary group remained similar to results presented for the whole athlete group.

Muscle protein expression. Muscle glucose uptake depends on the amount of GLUT4 in the muscle cell membrane. In sedentary individuals, membrane-bound GLUT4 was 28\% lower during Intralipid-insulin than during insulin and saline-insulin infusions $(1.23 \pm 0.10 \mathrm{AU}$ vs. $0.87 \pm 0.11 \mathrm{AU}, P<0.05)$. 

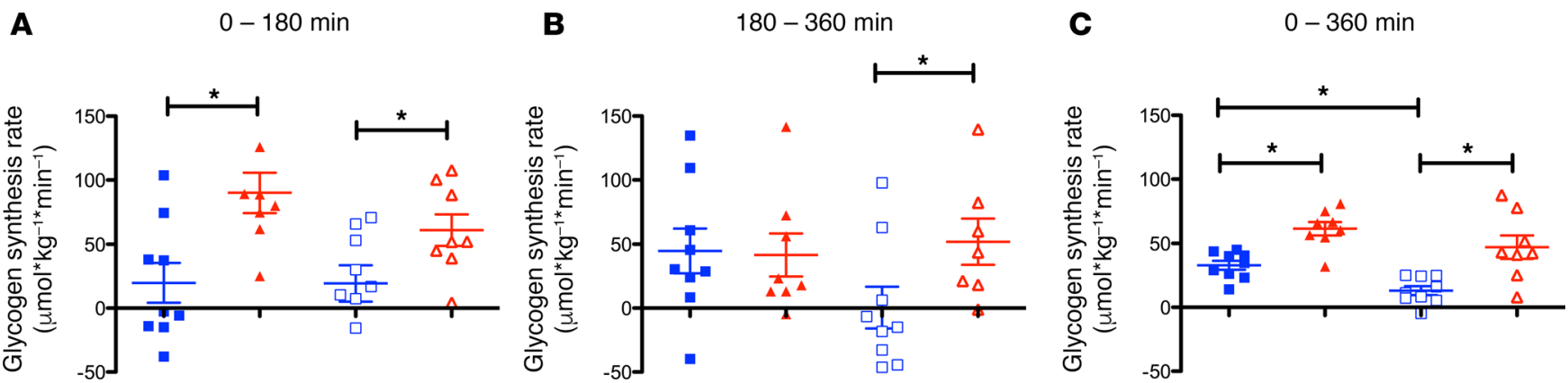

Figure 3. Rates of intramuscular glycogen synthesis during saline or Intralipid infusion. Nine sedentary humans (blue squares) and 8 endurance-trained athletes (red triangles) underwent 2 hyperinsulinemic-isoglycemic clamp tests with either saline (full symbols) or intralipid infusion (empty symbols). Rates of muscle glycogen synthesis are shown for the time intervals 0-180 minutes (A), 180-360 minutes (B), and 0-360 minutes (C) of the clamps. Data expressed as mean $\pm \mathrm{SEM} ;{ }^{*} P<0.05$.

In athletes, however, membrane-bound GLUT4 upon insulin stimulation was similar during the concomitant Intralipid or saline infusions (0.93 $\pm 0.18 \mathrm{AU}$ vs. $0.85 \pm 0.11 \mathrm{AU}, P=$ n.s., Figure 4$)$.

\section{Discussion}

This study reports for the first time to our knowledge the in vivo time course of intramyocellular G6P and glycogen concentrations in endurance-trained athletes under both low and high lipid availability. Although augmented lipid availability rapidly induces insulin resistance in sedentary humans at approximately 120 minutes - as reported before (8, 9, 20,33-35), endurance-trained athletes develop insulin resistance at approximately 180 minutes and thus are largely prevented from developing lipid-induced muscle insulin resistance by maintaining transmembrane muscle glucose transport.

By in vivo monitoring of glucose flux using multinuclei MRS, this study found that the lipid-induced insulin resistance in athletes is at least partly restored when compared with sedentary people. In line, muscle G6P levels in the athletes did not drop upon Intralipid infusion, and insulin-stimulated GSRs were maintained despite Intralipid infusion. This finding indicates that endurance training can protect against the inhibitory effect of fatty acids on glucose transport/phosphorylation with concomitant chronically high glycogen synthesis.

Muscle glycogen synthesis reflects NOGD and represents the major fraction of whole-body glucose disposal during insulin stimulation (13). The partial prevention of lipid-induced insulin resistance in the athletes seems mainly due to preserved NOGD. This finding extends our previous findings of higher wholebody NOGD in athletes than in sedentary humans during increased lipid availability (15). The remaining high NOGD supports the notion that myocellular glycogen synthesis could be the predominant route of whole-body insulin-stimulated NOGD in athletes also, as examined in the present study. The maintenance of high rates of glycogen synthesis could result either (a) from greater activity of glycogen synthase despite lower availability of G6P (resulting from impaired glucose transport/phosphorylation as reported for sedentary humans) or (b) from maintained glucose transport/phosphorylation resulting in constant myocellular G6P levels, sufficient to stimulate glycogen synthesis. Monitoring of myocellular G6P concentrations with ${ }^{31} \mathrm{P}$ MRS revealed marked differences between saline and Intralipid infusion. The decrease in G6P observed in the sedentary participants under lipid-infused conditions was consistent with the original report of lipid-induced effects on muscle glucose flux (9). In athletes, the absence of any decrease in G6P along with the constantly high GSRs despite high lipid availability clearly indicates that exercise training preserves myocellular glucose transport/phosphorylation under these conditions. These data are supported by a previous study demonstrating that severe insulin-resistant offspring of parents diagnosed with T2DM, featuring blunted insulin-stimulated increase of muscle G6P and reduced glycogen, exhibit a marked improvement of insulin-stimulated muscle G6P and glycogen synthesis upon chronic exercise training (36). Nevertheless, these studies show that exercise controls insulin-stimulated muscle glucose transport/phosphorylation and glycogen synthesis, thereby increasing insulin sensitivity. Of note, the improvement in insulin-stimulated whole-body glucose uptake after 12 weeks of exercise training seemed to be exclusively due to oxidative glucose metabolism, at least in T2DM and obese humans (37).

The athletes featured higher basal glycogen concentrations compared with the sedentary participants. By design, all participants refrained from exercise 3 days preceding the clamp tests. Actually, the 

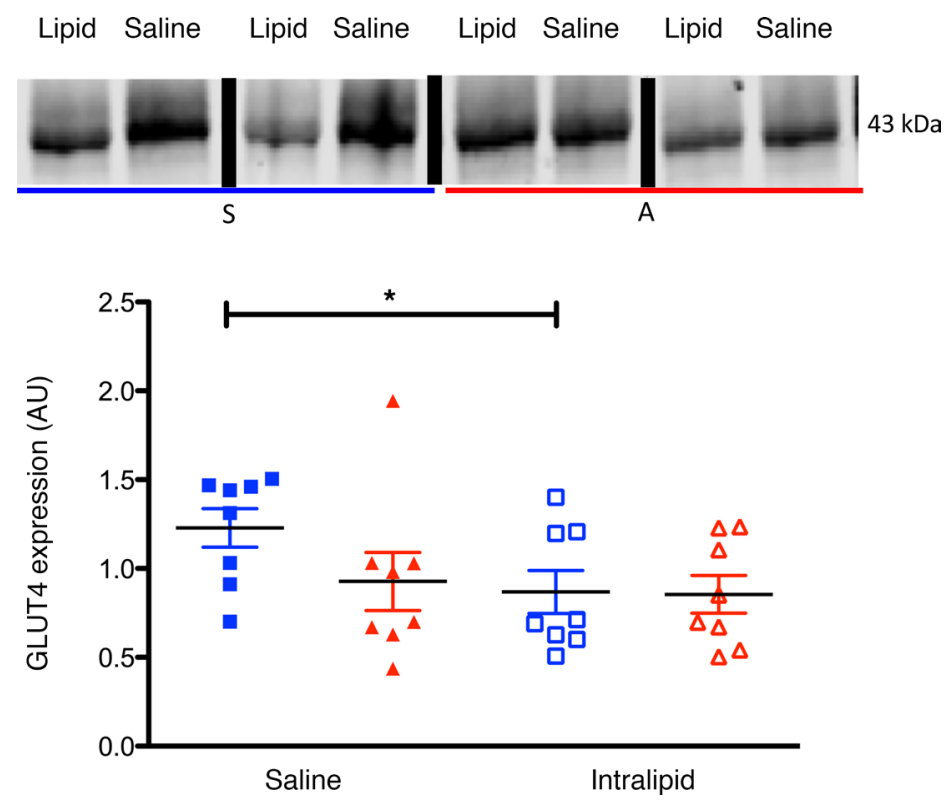

Figure 4. Expression of GLUT4 in skeletal muscle during saline or Intralipid infusion. Eight sedentary humans (blue squares) and 8 endurance-trained athletes (red triangles) underwent 2 hyperinsulinemic-isoglycemic clamp tests with either saline (full symbols) or Intralipid infusion (empty symbols). Biopsies were obtained from the vastus lateralis muscle at the end of the clamps. Lanes represent GLUT4 expression of 4 randomly chosen sedentary participants and athletes each, determined with Western blotting run on the same gel. The thick lines indicate noncontiguous lanes. Data expressed as mean $\pm \mathrm{SEM} ;{ }^{*} P<0.05$.

exercise constraint could explain the higher amount of glycogen stores because normally these glycogen stores would deplete upon exercise, depending on the exercise intensity and duration (38). However, lower glycogen content would stimulate activation of glycogen synthase and thereby drive muscle glycogen synthesis (39-41). In other words, the activity of glycogen synthesis has been shown to be greater when glycogen is heavily depleted. Previously, we reported that glycogen synthase phosphorylation before the start of the clamp tests is similar between athletes and sedentary participants (15). The higher baseline muscle glycogen levels in the athletes would therefore have not resulted in higher rates of glycogen synthesis than in the sedentary participants.

The maintained rates of muscle glucose uptake, as reflected by constant myocellular G6P, could have resulted from differences in myocellular lipid handling between athletes and sedentary participants. Our previous muscle biopsy study addressed myocellular TGs in vastus lateralis muscle (15). Briefly, only in type I, but not in type II fibers, the mean area fraction of TGs was increased exclusively in untrained persons upon exactly the same lipid infusion protocol as presented here. This is in line with previous results (42). In another study — although without an intralipid infusion protocol — trained athletes displayed lipid storage in a higher number of droplets in the intramyofibrillar region of type I fibers when compared with patients with T2DM, who store lipids predominately in larger droplets in the subsarcolemmal region of type II fibers (43). Together, these findings imply that lipid droplet location and distribution contribute to skeletal muscle insulin sensitivity.

The maintained rates of muscle glucose uptake in athletes could have resulted from greater glucose transport via GLUT4. Lipid infusion did not decrease membrane-bound GLUT4 content during insulin stimulation in athletes as compared to sedentary persons, exhibiting clearly compromised membrane-bound GLUT4 levels during Intralipid infusion. This likely contributes to the higher levels of muscle glucose transport in the athletes. Interestingly, one previous study found no alterations of insulin receptor signaling and AKT levels in athletes during lipid infusion (15). Muscle insulin resistance due to lipid infusion, obesity, or T2DM most likely occurs via activation of the DAG/PKC pathway, which leads to inhibitory serine phosphorylation of IRS-1 and subsequent reductions in AKT and glucose transport (30, 33). Consequently, the athletes' partial protection from insulin resistance may be the result of alteration in cellular lipid intermediates. A recent study provided supporting evidence by reporting a significant positive relationship 
between muscle PKC- $\varepsilon$ and sarcolemmal 1,2 DAG C16:0/C18:2 in insulin-resistant groups but not in athletes (31). In addition, lipid metabolites in other compartments may contribute to the metabolic changes: mitochondrial DAG accumulation has been hypothesized to promote increased mitochondrial morphology and function and thereby affect metabolism in athletes (44).

Previously, some studies reported that Intralipid infusion diminishes insulin-stimulated microvascular recruitment, which may contribute to reduced muscle glucose flux. Rodent studies support this concept by showing inhibition of insulin-mediated capillary recruitment by acute elevation of FFA (45). Studies in humans revealed inconsistent data. We found that vascular effects of Intralipid infusion are not rate controlling for human muscle insulin-stimulated glucose disposal (46), while others reported reduced insulin-stimulated microvascular recruitment upon lipid infusion $(47,48)$. The present study cannot rule out that the endurance-trained athletes were protected against this lipid-hampering of insulin-stimulated microvascular recruitment and in turn diminished glucose uptake.

In the present study, athletes featured a slightly higher basal rate of EGP than sedentary humans. The higher EGP may have contributed to the higher insulin-suppressed EGP percentage, which could have led to overestimation of the effect of exercise training on hepatic insulin sensitivity. Of note, knowledge on fasting and even postexercise hepatic glucose metabolism in endurance-trained athletes is still scarce. The current evidence indicates that - in contrast to skeletal muscle - liver glycogen concentrations as well glycogenolysis is lower in athletes than in untrained humans during moderate-to-high exercise (49). In the present study, both groups followed a carbohydrate-rich diet for 3 days preceding the clamp days, which would render such effects unlikely. Interestingly, prolonged low-carbohydrate diets may lead to lower EGP and glycogenolysis during exercise, when compared with a normal, mixed diet (50). One may speculate that the high basal EGP in athletes may result in increased responsiveness of the adrenal medulla, in turn favoring higher production and lower clearance of glucose (51). The design of the present study cannot rule out the operation of such a mechanism underlying the higher basal EGP values in chronically trained humans.

In this study, the athlete group consisted of both runners and cyclers. Although electromyography recording showed that both types of physical activity train the vastus lateralis muscle (52-54), some studies do report differences in the efficiency of the vastus lateralis muscle between runners and cyclers $(55,56)$. Separate analysis of glucose flux in runners and cyclers, however, revealed no differences between runners and cyclers.

Taken together, athletes exhibit higher muscle glucose disposal and glycogen synthesis during increased lipid availability primarily resulting from maintained insulin-stimulated glucose transport with increased myocellular G6P levels for subsequent glycogen synthesis.

\section{Methods}

Participants. Nine healthy, sedentary young humans (6 males $/ 3$ females) and 8 chronically endurance-trained humans ( 5 males $/ 3$ females), matched for age, were included (Table 1 and Supplemental Figure 1; supplemental material available online with this article; https://doi.org/10.1172/jci.insight.127928DS1). Medication use, family history of diabetes, and unstable dietary habits were exclusion criteria. All participants underwent an incremental aerobic cycling test until exhaustion (57). By design, endurance-trained humans had to participate in endurance exercise activities, such as running and/or cycling, thrice weekly for at least 2 years, with or without combination with other sports. Untrained participants had a sedentary lifestyle and did not participate in any regular physical exercise. Participants were defined as athletes if maximal oxygen uptake $\left(\mathrm{VO}_{2}\right.$ peak) was more than $55 \mathrm{~mL} / \mathrm{kg} / \mathrm{min}$ for men and more than $50 \mathrm{~mL} / \mathrm{kg} / \mathrm{min}$ for women and if insulin-stimulated $\mathrm{Rd}$ was more than $60 \mu \mathrm{mol} / \mathrm{kg} / \mathrm{min}$. Participants were defined as sedentary when $\mathrm{VO}_{2}$ peak was less than $45 \mathrm{~mL} / \mathrm{kg} / \mathrm{min}$ for both sexes. In total, 26 humans were screened, of whom 9 sedentary participants and 8 athletes fulfilled the inclusion criteria. All measurements were conducted at the research facility of the German Diabetes Center in Düsseldorf.

Hyperinsulinemic-euglycemic clamp. Three days before the clamp tests, all participants refrained from any exercise. Also, participants consumed a carbohydrate-rich meal the evening before the test day, i.e., fresh pasta with a minimum of approximately $115 \mathrm{~g}$ carbohydrates. On the test day, a baseline biopsy was taken from the vastus lateralis muscle before the clamp under local anesthesia ( $2 \%$ lidocaine) $(30,58)$. Each of the participants underwent two 6-hour hyperinsulinemic $\left(40 \mathrm{mU} / \mathrm{m}^{2} / \mathrm{min}\right)$ to euglycemic clamps with a primed, continuous $(0.04 \mathrm{mg} / \mathrm{kg} / \mathrm{min})$ intravenous infusion of $\left[6,6-{ }^{2} \mathrm{H}_{2}\right]$ glucose. Participants received an intravenous 
infusion of either $0.9 \%$ saline $(1.32 \mathrm{~mL} / \mathrm{min}$ as control condition $)$ or heparinized $(0.2 \mathrm{U} / \mathrm{kg} / \mathrm{min})$ long-chain triacylglycerols (1.32 mL/min; Intralipid, Braun, Melsungen, Germany) in random order $(9,15)$. Immediately after the clamp another muscle biopsy was taken.

MRS. The clamp tests were performed within a 3-T whole-body MRI scanner (Achieva Philips Healthcare, Best, the Netherlands). Participants were situated feet first into the magnet in the supine position, and the left leg was placed in an MRI-compatible foot brace, which was secured firmly with straps for the in vivo detection of G6P and glycogen concentrations within the vastus lateralis muscle. Real-time monitoring of G6P and glycogen concentrations was conducted at baseline, and during $t$ $=60,120,180,240,300,360$ minutes of the clamp, via individual $7-\mathrm{cm}{ }^{31} \mathrm{P}$ and ${ }^{13} \mathrm{C}$ coils equipped with 12-cm quadrature ${ }^{1} \mathrm{H}$ coils for decoupling, shimming, and imaging (PulseTeq, Cobham, United Kingdom). All MRS spectra were processed within the NUTS software package (Acorn NMR Inc., Livermore, California, USA). ${ }^{31} \mathrm{P}-\mathrm{MRS}$ proton-decoupled pulse-acquired measurements (repetition time [TR]: $3500 \mathrm{~ms}$, bandwidth [BW]: $2048 \mathrm{kHz}$, number of averages [NSA]: 100, decoupling: WALTZ-4, time: 6 minutes) were conducted with an excitation block pulse calibrated to adjust for coil loading via multiple flip angle measurements of an internal $\mathrm{H}_{3} \mathrm{PO}_{4}$ reference to provide $90^{\circ}$ excitation at $2 \mathrm{~cm}$. Differences in G6P spectra (effective line broadening: $10 \mathrm{~Hz}$, zero filling: 32k) were detected through integration of the region between 6.83 and 7.43 parts per million (ppm) (12) after the subtraction of spectra from baseline measurements and baseline correction. Concentrations of G6P were determined through comparing the resonance areas with the $\beta$-ATP resonance from a fully relaxed (TR: 15 seconds) baseline spectrum, and a concentration of $5.5 \mathrm{mmol} / \mathrm{kg}$ muscle, as assumed for resting ATP concentration (59) ${ }^{13} \mathrm{C}-\mathrm{MRS}$ proton-decoupled pulse-acquired measurements (TR: $197 \mathrm{~ms}, \mathrm{BW}: 8 \mathrm{kHz}$, NSA: 4000, data points: 256, decoupling: continuous wave, time: 13 minutes) were acquired with a 4.992-ms rapid half-passage adiabatic excitation pulse. Coil loading was corrected via integration of the rightmost peak of a ${ }^{13} \mathrm{C}$-enriched sample of formic acid (MilliporeSigma, Milwaukee, Wisconsin, USA) placed within the coil housing (60). C1-glycogen peak areas were determined through the integration of the region between 85 and 115 ppm after signal conditioning (effective line broadening: $20 \mathrm{~Hz}$, zero filling: 8k) and baseline correction. Absolute concentrations of glycogen within the vastus lateralis muscle were determined from 1-L aqueous phantom replacement measurements $(\mathrm{KCl}: 40 \mathrm{mM}$, sodium azide: 0.05\%, phosphocreatine: $40 \mathrm{mM}$, glycogen: 30, 60, and $90 \mathrm{mM}$, MilliporeSigma, Milwaukee, Wisconsin, USA), and correction for B1-field penetration depth $(0-30 \mathrm{~mm})$ via agar gel spacers (KCl: $70 \mathrm{mM}$, agar: $2 \%$ weight/volume) was used to simulate subcutaneous adipose tissue.

Blood analyses. Blood samples were immediately cooled and centrifuged and supernatants stored at $-80^{\circ} \mathrm{C}$. Blood glucose was measured using the glucose oxidase method (EKF biosen C-Line glucose analyzer, EKF Diagnostics GmbH, Barleben, Germany) (61). Serum TGs were analyzed enzymatically (Hitachi analyzer, Roche Diagnostics, Mannheim, Germany) and FFAs microfluorimetrically (Wako, Neuss, Germany) after prevention of lipolysis using orlistat (62). Plasma glycerol was measured enzymatically (R-Biopharm, Darmstadt, Germany). Isotopic enrichments of plasma glucose were measured as previously described (63).

Western blotting. For Western blot analysis, equal amounts of protein were loaded and protein concentration was directly controlled and analyzed on the nitrocellulose blots by the REVERT Total Protein Stain Kit (LI-COR Biosciences, Westburg, Leusden, the Netherlands). For GLUT4 content, muscle membrane fractions and total muscle protein fractions were prepared as described previously (64). Blots for GLUT4 were incubated with a goat polyclonal GLUT4 antibody (sc1608; Santa Cruz Biotechnology, BioConnect, Huissen, the Netherlands). An appropriate near-infrared-tagged secondary antibody (IRDye, LI-COR Biosciences, Westburg, Leusden, the Netherlands) was used to visualize and quantify the appreciated GLUT4 band with the Odyssey near-infrared imaging system (LI-COR Biosciences, Westburg, Leusden, the Netherlands). Because of technical issues, the GLUT4 expression analysis was performed in $n=8$ athletes and $n=6$ sedentary humans. See the full, uncut blots in the online supplemental materials.

Calculations. Ra and Rd were calculated for non-steady state conditions according to Steele (65). Peripheral insulin sensitivity was expressed as the $\Delta \mathrm{Rd}$, which represents the difference between insulin-stimulated and baseline Rd. EGP was calculated from the difference between Ra and mean GIRs. Hepatic insulin sensitivity was calculated as percentage of EGP suppression by insulin during clamp steady state versus baseline (EGP). Net GSR was calculated from the slope of the linear fit to the glycogen concentration time curve during the periods 0-180 minutes and 180-360 minutes (9). The development of intralipid-induced insulin resistance occurs between 120 and 180 minutes in sedentary people $(9,34,35)$, which forms the 
rationale for splitting the analysis of GSR into these 2 time intervals. For the athletes so far, no information on the time course of lipid-induced insulin resistance has been reported. The GSR, however, was also calculated over the entire period (0-360 minutes). For the GIR and both the time courses of $\Delta$ glycogen and $\triangle \mathrm{G} 6 \mathrm{P}$ concentrations, the AUC was calculated using the trapezoid method. Test days were randomized by the first authors of this study using https://www.randomizer.org.

Statistics. Statistical analyses were performed using the statistics computer program SPSS 18 for Mac OS X. Differences between groups and between conditions were analyzed by 2-way ANOVA, in which comparisons between groups for repeated measures were computed with Tukey's post hoc correction. Pearson's linear regression analysis was performed to identify correlations between variables. Differences were considered significant at $P<0.05$.

Study approval. The institutional ethics board of Heinrich-Heine University approved the study, and all participants gave their informed consent in writing.

\section{Author contributions}

EP, MR, MKCH, and PS designed the study. EP, PB, SG, AB, EK, and GS contributed to the data collection and/or analysis. EP, PB, and SG performed the statistical analysis. All authors contributed to the interpretation of the results. EP and MR wrote the manuscript. All authors revised it critically for important intellectual content and approved the submission of the final version.

\section{Acknowledgments}

The authors appreciate the voluntary contribution of all study participants. The authors also thank Kai Tinnes and Myrko Esser (German Diabetes Center [DDZ], Düsseldorf, Germany) for excellent technical assistance and taking care of the patients. The authors further thank Irena Latta (DDZ, Düsseldorf, Germany) for excellent technical assistance. MR is the guarantor of this work and, as such, had full access to all the data in the study and takes responsibility for the integrity of the data and the accuracy of the data analysis.

The German Diabetes Center is funded by the BMG and the Ministry of Culture and Science of the State North Rhine-Westphalia. This study was also supported in part by grants from the German Federal Ministry of Education and Research (BMBF) to the German Center for Diabetes Research (DZD e.V.), from the German Research Foundation (DFG) through the Collaborative Research Center SFB 1116 (Master switches in cardiac ischemia), and from the Schmutzler Stiftung. The funders had no role in study design, data collection, data analysis, data interpretation, writing of the report, or decision to publish the manuscript.

Address correspondence to: Michael Roden, Division of Endocrinology and Diabetology, Medical Faculty, Heinrich-Heine University, Auf'm Hennekamp 65, 40225 Düsseldorf, Germany. Phone: 49.211.3382.201; Email: michael.roden@ddz.uni-duesseldorf.de.

1. Samuel VT, Shulman GI. The pathogenesis of insulin resistance: integrating signaling pathways and substrate flux. J Clin Invest. 2016;126(1):12-22.

2. Tilg H, Moschen AR, Roden M. NAFLD and diabetes mellitus. Nat Rev Gastroenterol Hepatol. 2017;14(1):32-42.

3. Krssak M, et al. Intramyocellular lipid concentrations are correlated with insulin sensitivity in humans: a 1H NMR spectroscopy study. Diabetologia. 1999;42(1):113-116.

4. Bachmann OP, et al. Effects of intravenous and dietary lipid challenge on intramyocellular lipid content and the relation with insulin sensitivity in humans. Diabetes. 2001;50(11):2579-2584.

5. Perseghin G, et al. Normal insulin sensitivity and IMCL content in overweight humans are associated with higher fasting lipid oxidation. Am J Physiol Endocrinol Metab. 2002;283(3):E556-E564.

6. Petersen MC, Shulman GI. Roles of diacylglycerols and ceramides in hepatic insulin resistance. Trends Pharmacol Sci. 2017;38(7):649-665.

7. Roden M. How free fatty acids inhibit glucose utilization in human skeletal muscle. News Physiol Sci. 2004;19:92-96.

8. Yu C, et al. Mechanism by which fatty acids inhibit insulin activation of insulin receptor substrate-1 (IRS-1)-associated phosphatidylinositol 3-kinase activity in muscle. J Biol Chem. 2002;277(52):50230-50236.

9. Roden M, et al. Mechanism of free fatty acid-induced insulin resistance in humans. J Clin Invest. 1996;97(12):2859-2865.

10. Roden M, et al. Rapid impairment of skeletal muscle glucose transport/phosphorylation by free fatty acids in humans. Diabetes. 1999;48(2):358-364

11. Rothman DL, et al. Decreased muscle glucose transport/phosphorylation is an early defect in the pathogenesis of non-insulin-dependent diabetes mellitus. Proc Natl Acad Sci USA. 1995;92(4):983-987.

12. Rothman DL, Shulman RG, Shulman GI. 31P nuclear magnetic resonance measurements of muscle glucose-6-phosphate. 
Evidence for reduced insulin-dependent muscle glucose transport or phosphorylation activity in non-insulin-dependent diabetes mellitus. J Clin Invest. 1992;89(4):1069-1075.

13. Shulman GI, Rothman DL, Jue T, Stein P, DeFronzo RA, Shulman RG. Quantitation of muscle glycogen synthesis in normal subjects and subjects with non-insulin-dependent diabetes by 13C nuclear magnetic resonance spectroscopy. N Engl J Med. 1990;322(4):223-228.

14. Goodpaster BH, He J, Watkins S, Kelley DE. Skeletal muscle lipid content and insulin resistance: evidence for a paradox in endurance-trained athletes. J Clin Endocrinol Metab. 2001;86(12):5755-5761.

15. Phielix E, et al. High oxidative capacity due to chronic exercise training attenuates lipid-induced insulin resistance. Diabetes. 2012;61(10):2472-2478.

16. Roden M, Petersen K, Shulman G. Insulin resistance in type 2 diabetes. In: Holt RIG, Cockram C, Flyvbjerg A, Goldstein BJ, eds. Textbook of Diabetes. 5th ed. Hoboken, New Jersey, USA: Wiley-Blackwell; 2017:174-186.

17. Ciaraldi TP, Abrams L, Nikoulina S, Mudaliar S, Henry RR. Glucose transport in cultured human skeletal muscle cells. Regulation by insulin and glucose in nondiabetic and non-insulin-dependent diabetes mellitus subjects. J Clin Invest. 1995;96(6):2820-2827.

18. Garvey WT, Maianu L, Zhu JH, Brechtel-Hook G, Wallace P, Baron AD. Evidence for defects in the trafficking and translocation of GLUT4 glucose transporters in skeletal muscle as a cause of human insulin resistance. J Clin Invest. 1998;101(11):2377-2386.

19. Cline GW, et al. Impaired glucose transport as a cause of decreased insulin-stimulated muscle glycogen synthesis in type 2 diabetes. N Engl J Med. 1999;341(4):240-246.

20. Dresner A, et al. Effects of free fatty acids on glucose transport and IRS-1-associated phosphatidylinositol 3-kinase activity. J Clin Invest. 1999;103(2):253-259.

21. Dela F, et al. Physical training increases muscle GLUT4 protein and mRNA in patients with NIDDM. Diabetes. 1994;43(7):862-865.

22. Christ-Roberts CY, et al. Exercise training increases glycogen synthase activity and GLUT4 expression but not insulin signaling in overweight nondiabetic and type 2 diabetic subjects. Metab Clin Exp. 2004;53(9):1233-1242.

23. Richter EA, et al. Training effects on muscle glucose transport during exercise. Adv Exp Med Biol. 1998;441:107-116.

24. Kraniou GN, Cameron-Smith D, Hargreaves M. Acute exercise and GLUT4 expression in human skeletal muscle: influence of exercise intensity. J Appl Physiol. 2006;101(3):934-937.

25. Schenk S, Horowitz JF. Acute exercise increases triglyceride synthesis in skeletal muscle and prevents fatty acid-induced insulin resistance. J Clin Invest. 2007;117(6):1690-1698.

26. Tsintzas K, Chokkalingam K, Jewell K, Norton L, Macdonald IA, Constantin-Teodosiu D. Elevated free fatty acids attenuate the insulin-induced suppression of PDK4 gene expression in human skeletal muscle: potential role of intramuscular long-chain acyl-coenzyme A. J Clin Endocrinol Metab. 2007;92(10):3967-3972.

27. Storgaard $\mathrm{H}$, et al. Dissociation between fat-induced in vivo insulin resistance and proximal insulin signaling in skeletal muscle in men at risk for type 2 diabetes. J Clin Endocrinol Metab. 2004;89(3):1301-1311.

28. Høeg LD, et al. Lipid-induced insulin resistance affects women less than men and is not accompanied by inflammation or impaired proximal insulin signaling. Diabetes. 2011;60(1):64-73.

29. Hoy AJ, et al. Lipid and insulin infusion-induced skeletal muscle insulin resistance is likely due to metabolic feedback and not changes in IRS-1, Akt, or AS160 phosphorylation. Am J Physiol Endocrinol Metab. 2009;297(1):E67-E75.

30. Szendroedi J, et al. Role of diacylglycerol activation of PKC $\theta$ in lipid-induced muscle insulin resistance in humans. Proc Natl Acad Sci USA. 2014;111(26):9597-9602.

31. Perreault L, et al. Intracellular localization of diacylglycerols and sphingolipids influences insulin sensitivity and mitochondrial function in human skeletal muscle. JCI Insight. 2018;3(3):96805.

32. Metges CC, et al. Kinetics of L-[1-(13)C]leucine when ingested with free amino acids, unlabeled or intrinsically labeled casein. Am J Physiol Endocrinol Metab. 2000;278(6):E1000-E1009.

33. Itani SI, Ruderman NB, Schmieder F, Boden G. Lipid-induced insulin resistance in human muscle is associated with changes in diacylglycerol, protein kinase C, and IkappaB-alpha. Diabetes. 2002;51(7):2005-2011.

34. Boden G, Chen X, Ruiz J, White JV, Rossetti L. Mechanisms of fatty acid-induced inhibition of glucose uptake. J Clin Invest. 1994;93(6):2438-2446.

35. Boden G, et al. Effects of fat on insulin-stimulated carbohydrate metabolism in normal men. J Clin Invest. 1991;88(3):960-966.

36. Perseghin G, et al. Increased glucose transport-phosphorylation and muscle glycogen synthesis after exercise training in insulin-resistant subjects. $N$ Engl J Med. 1996;335(18):1357-1362.

37. Meex RC, et al. Restoration of muscle mitochondrial function and metabolic flexibility in type 2 diabetes by exercise training is paralleled by increased myocellular fat storage and improved insulin sensitivity. Diabetes. 2010;59(3):572-579.

38. Price TB, Rothman DL, Avison MJ, Buonamico P, Shulman RG. 13C-NMR measurements of muscle glycogen during low-intensity exercise. J Appl Physiol. 1991;70(4):1836-1844.

39. Maehlum S, Hermansen L. Muscle glycogen concentration during recovery after prolonged severe exercise in fasting subjects. Scand J Clin Lab Invest. 1978;38(6):557-560

40. Maehlum S, Høstmark AT, Hermansen L. Synthesis of muscle glycogen during recovery after prolonged severe exercise in diabetic and non-diabetic subjects. Scand J Clin Lab Invest. 1977;37(4):309-316.

41. Ivy JL. Muscle glycogen synthesis before and after exercise. Sports Med. 1991;11(1):6-19.

42. Chow LS, Mashek DG, Wang Q, Shepherd SO, Goodpaster BH, Dubé JJ. Effect of acute physiological free fatty acid elevation in the context of hyperinsulinemia on fiber type-specific IMCL accumulation. J Appl Physiol. 2017;123(1):71-78.

43. Daemen S, et al. Distinct lipid droplet characteristics and distribution unmask the apparent contradiction of the athlete's paradox. Mol Metab. 2018;17:71-81.

44. Amati F, et al. Skeletal muscle triglycerides, diacylglycerols, and ceramides in insulin resistance: another paradox in endurance-trained athletes? Diabetes. 2011;60(10):2588-2597.

45. Clerk LH, Rattigan S, Clark MG. Lipid infusion impairs physiologic insulin-mediated capillary recruitment and muscle glucose uptake in vivo. Diabetes. 2002;51(4):1138-1145.

46. Szendroedi J, et al. Lipid-induced insulin resistance is not mediated by impaired transcapillary transport of insulin and glucose in humans. Diabetes. 2012;61(12):3176-3180.

47. Liu J, Jahn LA, Fowler DE, Barrett EJ, Cao W, Liu Z. Free fatty acids induce insulin resistance in both cardiac and skeletal 
muscle microvasculature in humans. J Clin Endocrinol Metab. 2011;96(2):438-446.

48. de Jongh RT, Serné EH, Ijzerman RG, de Vries G, Stehouwer CD. Free fatty acid levels modulate microvascular function: relevance for obesity-associated insulin resistance, hypertension, and microangiopathy. Diabetes. 2004;53(11):2873-2882.

49. Gonzalez JT, Fuchs CJ, Betts JA, van Loon LJ. Liver glycogen metabolism during and after prolonged endurance-type exercise. Am J Physiol Endocrinol Metab. 2016;311(3):E543-E553.

50. Webster CC, Noakes TD, Chacko SK, Swart J, Kohn TA, Smith JA. Gluconeogenesis during endurance exercise in cyclists habituated to a long-term low carbohydrate high-fat diet. J Physiol (Lond). 2016;594(15):4389-4405.

51. Kjaer M, Christensen NJ, Sonne B, Richter EA, Galbo H. Effect of exercise on epinephrine turnover in trained and untrained male subjects. J Appl Physiol. 1985;59(4):1061-1067.

52. Montgomery WH, Pink M, Perry J. Electromyographic analysis of hip and knee musculature during running. Am J Sports Med. 1994;22(2):272-278

53. Ericson MO, Nisell R, Arborelius UP, Ekholm J. Muscular activity during ergometer cycling. Scand J Rehabil Med. 1985;17(2):53-61.

54. Bohm S, Marzilger R, Mersmann F, Santuz A, Arampatzis A. Operating length and velocity of human vastus lateralis muscle during walking and running. Sci Rep. 2018;8(1):5066.

55. Bijker KE, de Groot G, Hollander AP. Differences in leg muscle activity during running and cycling in humans. Eur J Appl Physiol. 2002;87(6):556-561.

56. Bijker KE, De Groot G, Hollander AP. Delta efficiencies of running and cycling. Med Sci Sports Exerc. 2001;33(9):1546-1551.

57. Kuipers H, Verstappen FT, Keizer HA, Geurten P, van Kranenburg G. Variability of aerobic performance in the laboratory and its physiologic correlates. Int J Sports Med. 1985;6(4):197-201.

58. Phielix E, Jelenik T, Nowotny P, Szendroedi J, Roden M. Reduction of non-esterified fatty acids improves insulin sensitivity and lowers oxidative stress, but fails to restore oxidative capacity in type 2 diabetes: a randomised clinical trial. Diabetologia. 2014;57(3):572-581.

59. Harris RC, Hultman E, Nordesjö LO. Glycogen, glycolytic intermediates and high-energy phosphates determined in biopsy samples of musculus quadriceps femoris of man at rest. Methods and variance of values. Scand J Clin Lab Invest. 1974;33(2):109-120.

60. Hernández EÁ, et al. Acute dietary fat intake initiates alterations in energy metabolism and insulin resistance. J Clin Invest. 2017;127(2):695-708.

61. Nowotny B, Nowotny PJ, Strassburger K, Roden M. Precision and accuracy of blood glucose measurements using three different instruments. Diabet Med. 2012;29(2):260-265.

62. Brehm A, Krssak M, Schmid AI, Nowotny P, Waldhäusl W, Roden M. Increased lipid availability impairs insulin-stimulated ATP synthesis in human skeletal muscle. Diabetes. 2006;55(1):136-140.

63. Stingl H, et al. Lipid-dependent control of hepatic glycogen stores in healthy humans. Diabetologia. 2001;44(1):48-54.

64. Borghouts LB, Schaart G, Hesselink MK, Keizer HA. GLUT-4 expression is not consistently higher in type-1 than in type-2 fibres of rat and human vastus lateralis muscles; an immunohistochemical study. Pflugers Arch. 2000;441(2-3):351-358.

65. Steele R. Influences of glucose loading and of injected insulin on hepatic glucose output. Ann N Y Acad Sci. 1959;82:420-430. 